Research Paper

\title{
Knockdown of Tubulin Polymerization Promoting Protein Family Member 3 Suppresses Proliferation and Induces Apoptosis in Non-Small-Cell Lung Cancer
}

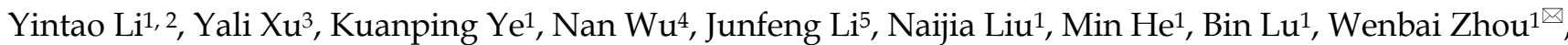 \\ Renming $\mathrm{Hu}^{1 凶}$ \\ 1. Department of Endocrinology and Metabolism, Institute of Endocrinology and Diabetology, Huashan Hospital, Fudan University, Shanghai, P.R. China \\ 2. Department of Medical Oncology, Shandong Cancer Hospital and Institute, Jinan, Shandong, P.R. China \\ 3. Department of Pathology, Provincial Hospital Affiliated to Shandong University, Jinan, Shandong, P.R. China \\ 4. Department of Geriatrics, Zhongshan Hospital, Fudan University, Shanghai, P.R. China \\ 5. Department of Endocrinology, Renmin Hospital of Wuhan University, Wuhan, Hubei, P.R. China. \\ $\triangle$ Corresponding authors: zhouwenbai@hotmail.com (W. Zhou), renminghu@fudan.edu.cn (R. Hu).
}

(1) Ivyspring International Publisher. Reproduction is permitted for personal, noncommercial use, provided that the article is in whole, unmodified, and properly cited. See http://ivyspring.com/terms for terms and conditions.

Received: 2015.12.23; Accepted: 2016.04.26; Published: 2016.06.18

\begin{abstract}
Our previous studies demonstrated that depletion of tubulin polymerization promoting protein family member 3 (TPPP3) inhibits proliferation and induces apoptosis of HeLa cells. However, the expression and roles of TPPP3 in cancers remain largely unknown. In this study, we investigated the expression of TPPP3 in clinicopathological correlations in non-small-cell lung cancer (NSCLC) samples by immunohistochemistry. TPPP3 expression was significantly upregulated in NSCLC tissues, and high TPPP3 expression was positively associated with tumor size, lymph node metastasis, clinical stage, and poor survival. Furthermore, knockdown of TPPP3 by shRNA significantly inhibited cell proliferation and induced cell apoptosis and cell cycle arrest in vitro. In addition, depletion of TPPP3 inhibited lung cancer growth in vivo in the xenografts of $\mathrm{H} 1299$ cells; this effect was accompanied by the suppression of Ki67 expression. Our data suggested that TPPP3 might act as an oncogene in NSCLC. TPPP3 warrants consideration as a therapeutic candidate with anti-tumor potential.
\end{abstract}

Key words: TPPP3, NSCLC, cell proliferation, cell apoptosis.

\section{Introduction}

Lung cancer is the leading cause of cancer-related death worldwide, with approximately 1.4 to 1.6 million deaths per year [1]. Approximately 75 to $80 \%$ of lung cancer is classified as NSCLC, including adenocarcinoma, squamous cell carcinoma, adenosquamous cell carcinoma and large cell carcinoma [2]. Despite rapid treatment advances, including radical resection, chemotherapy and targeted therapy, the five-year survival rate remains less than $16 \%$ worldwide [3]. Thus, to investigate the molecular mechanisms underlying the pathogenesis of NSCLC, it is necessary to identify novel targets for curative therapies.

Microtubule dynamics are modulated by several accessory proteins named microtubule-associated proteins (MAPs), most of which serve as the positive regulators of MT assembly either by promoting the tubulin polymerization or by stabilizing MTs $[4,5]$. MAPs can significantly influence the kinetics of microtubule nucleation, acting either as nucleation-promoting or nucleation-inhibiting factors [6]. Tubulin polymerization promoting proteins (TPPPs), including TPPP1, TPPP2 and TPPP3, have recently been identified as a new eukaryotic protein family $[7,8]$. These proteins exhibit MAP-like function by causing MT bundling and stabilizing the microtubular network $[9,10]$. TPPP1 promotes tubulin assemblies, blocks mitotic spindle formation and affects cell proliferation [11]. TPPP3, a member of the TPPP protein family, was first cloned by gene 
expression profiling in the human hypothalamus-pituitary-adrenal axis [8]. TPPP3 is a specific marker of the developing musculoskeletal system though inducing tubulin polymerization [12]. TPPP3 expression is increased in lung cancer and/or COPD groups, indicating that it may be a potential target for the early diagnosis of lung cancer [13]. Our previous study demonstrated that knockdown of TPPP3 by RNAi inhibits proliferation and induces cell cycle arrest in HeLa cells and suppresses tumor growth and metastasis in Lewis lung carcinoma cells $[5,14]$. However, little is known about the expression pattern of TPPP3 in lung cancer tissues, and the biological functions of TPPP3 remain largely unknown.

The aim of this study was to further investigate the expression and function of TPPP3 protein in vitro and in vivo and to elucidate potential molecular mechanisms. In this study, we observed that TPPP3 was upregulated in NSCLC tissues and associated with reduced overall survival. Furthermore, knockdown of TPPP3 inhibited cell proliferation and induced cell apoptosis and cell cycle arrest in vitro. Finally, we demonstrated that depletion of TPPP3 suppressed tumor growth in a nude mouse xenograft model.

\section{Materials and methods}

\section{Tissue samples and clinical data}

The paraffin-embedded lung cancer tissue microarray containing 76 paired samples (152 spots, including both tumor and adjacent normal tissues) was obtained from Shanghai Biochip Co. Ltd (Shanghai). All patients were confirmed by pathological evaluation. None of these patients received any preoperative adjuvant therapy. Written informed consent was obtained from each patient. This study was approved by the Medical Ethics Committee of Huashan Hospital, Fudan University.

\section{Cell lines and cell culture}

Human embryonic kidney 293T cells (HEK293T), a normal human bronchial cell line (HBE), and human NSCLC cell lines (95C, SPC-A1, A549, and H1299) were purchased from cell banks of the Shanghai Institutes of Biological Sciences (Shanghai, China). HEK293T and HBE were cultured in DMEM medium. In addition, 95C, SPC-A1, A549, and H1299 were cultured in RPMI-1640 medium. All of the media were supplemented with $10 \%$ fetal bovine serum (Invitrogen, Carlsbad, CA, USA) and 1\% penicillin/streptomycin at $37^{\circ} \mathrm{C}$ with $5 \% \mathrm{CO}_{2}$ and $95 \%$ humidity.

\section{Immunohistochemistry}

Immunohistochemical staining was performed with the standard approach as previously described [15]. In brief, tissue microarray slides were deparaffinized in xylene and rehydrated through an ethanol gradient $(100 \%, 95 \%, 90 \%, 80 \%$, and $70 \%$, each for $5 \mathrm{~min}$ ). The antigen was retrieved by citric acid buffer ( $\mathrm{pH}$ 6.0), and endogenous peroxidase activity was blocked by 3\% $\mathrm{H} 2 \mathrm{O} 2$. Following blocking with normal goat serum, the slide was incubated with rabbit anti-human TPPP3 primary polyclonal antibody (ABGENT, China) overnight in a humidified container at $4^{\circ} \mathrm{C}$. Polyperoxidase-anti-rabbit IgG was incubated according to the manufacturer's recommendations (DAKO, Denmark, CA, USA).

Two independent pathologists who were blind to the patients' clinicopathological features evaluated the immunostaining score. The staining intensity was scored as 0 (negative), 1 (weak), 2 (moderate), and 3 (strong). The proportion of positive tumor cells was assigned as 0 ( $<25 \%$ positive), 1 ( $25-50 \%$ positive), 2 (50-75\% positive), and 3 (75-100\% positive). The final score was calculated by multiplying the staining intensity score and the percentage of positively stained tumor cells. The patients were divided into two groups (low TPPP3 and high TPPP3) according to median TPPP3 expression in cancer samples.

\section{Quantitative real-time PCR analysis}

Total RNA was isolated from tissues and cells using Trizol reagent (Life Technologies) according to the manufacturer's protocols. For reverse transcripttion, $1 \mu \mathrm{g}$ of the total RNA was converted to first strand complementary DNA in $20-\mu l$ reactions using a cDNA synthesis kit (KAPA Biosystems, Woburn, MA, USA). The relative mRNA level was determined by quantitative real-time PCR using the SYBR Green Master Mix Kit (KAPA Biosystems) on an ABI 7500 Real-Time PCR system (Applied Biosystems). The following primers were used: TPPP3, 5'-GGTCCATTCCTGCGTCGTTC-3' (forward) and 5'-GCCCAGTTCTTGCCATTCATC-3' (reverse); $\beta$-actin, 5'-CATGTACGTTGCTATCCAGGC-3' (forward) and 5'-CTCCTTAATGTCACGCACGAT-3' (reverse). $\beta$-actin was used as an internal control, and the relative expression of TPPP3 was normalized to $\beta$-actin.

\section{Small hairpin RNA (shRNA) lentiviruses}

shRNAs targeting TPPP3 were designed and constructed by Shanghai Genechem (Shanghai, China). The following sequences were inserted into the GV112 vector to form TPPP3 shRNAs: sh1: 5'-CCGGGCCAATGTGGGCGTCACTAAACTCGAG TTTAGTGACGCCCACATTGGCTTTTTG-3', sh2: 
5'-CCGGCTGCTCGGGTCATCAACTATGCTCGAG CATAGTTGATGACCCGAGCAGTTTTTG-3', sh3: 5'-CCGGAGGAGAGCTTCCGCAAGTTTGCTCGAG CAAACTTGCGGAAGCTCTCCTTTTTTG-3.

HEK293T cells were cotransfected with the lentivirus-mediated control vector (SCR) or shTPPP3, Lentivector Packaging KIT (Genechem) using TurboFect $^{\mathrm{TM}}$ (Fermentas, Glen Burnie, MD, USA) according to the manufacturer's instructions. Cells were transduced with SCR or ShTPPP3, and the efficiency of knockdown was measured $96 \mathrm{~h}$ after cells were infected.

\section{Cell proliferation assay and colony formation}

Cell proliferation was assessed by the cell CCK-8 Assay (Dojindo Lab, Kumamoto, Japan) according to the manufacturer's protocol. Cells in a 96-well plate were incubated with CCK-8 solutions at different times for $2 \mathrm{~h}$ at $37^{\circ} \mathrm{C}$. Absorbance of each well was quantified at $450 \mathrm{~nm}$ by the Infinite $200 \mathrm{PRO}$ Microplate Reader (Tecan, Mannedorf, Switzerland).

For the colony formation assay, 1200 cells were plated into each well of six-well plates. Medium was refreshed every three days. Colonies were stained with $1 \%$ crystal violet for 15 minutes 14 days later and counted by visual inspection.

\section{Flow cytometry analysis}

For apoptosis analysis, cells were harvested at a density of $2 \times 10^{5}$ cells $/ \mathrm{ml}$ in $1 \times$ binding buffer and stained with the Annexin V/propidium iodide (PI) staining assay (BD Biosciences, San Jose, CA, USA) for $15 \mathrm{~min}$ at room temperature and then analyzed using a FACSCalibur flow cytometer and CellQuest software (BD Biosciences).

For cell cycle analysis, cells were harvested by trypsinization and washed with ice-cold PBS. Following fixation in $75 \%$ alcohol at $4^{\circ} \mathrm{C}$ overnight, cells were treated with RNAse (Sigma, Saint Louis, MI, USA) for $15 \mathrm{~min}$ at $37^{\circ} \mathrm{C}$ and then stained with PI (Sigma) for $15 \mathrm{~min}$ in the dark room. DNA content analysis was performed using a FACSCalibur flow cytometer and CellQuest software (BD Biosciences) by collecting 10,000 events for the cell cycle.

\section{Western blot analysis}

Cell protein was extracted using RIPA lysis buffer (Cell Signaling Technology, Danvers, MA, USA) and complete protease inhibitor cocktail (Roche Diagnostics) at $4^{\circ} \mathrm{C}$. Protein was separated by $10 \%$ SDS-PAGE and then transferred to $0.45-\mu \mathrm{m}$ PVDF membranes (Bio-Rad, CA, USA). After blocking using $5 \%$ non-fat milk, membranes were incubated with AKT, p-AKT (ser473), STAT3, p-STAT3 (ser727), Survivin, Cyclind1, and Bcl-2 (all from Cell Signaling Technology) antibodies overnight at $4^{\circ} \mathrm{C}$ followed by incubation with the appropriate HRP-conjugated secondary antibody for $1 \mathrm{~h}$ at room temperature. Blotted membranes were stripped and re-blotted with a $\beta$-actin monoclonal antibody (Sigma) as a loading control. Blots were detected using enhanced chemiluminescence (ECL) detection systems (Pierce, Rockford, IL, USA). Bands densitometry were analyzed by Image J software (National Institutes of Health, USA).

\section{Animal experiments}

The study was performed in accordance with the guidelines for animal experiments of Fudan University (permit number of 20141026QY). Four-week-old female BALB/c-nude mice were purchased from the Animal Center of the Chinese Academy of Science (Shanghai, China). Briefly, $1 \times 10^{7}$ H1299 cells transduced with SCR or shTPPP3 were subcutaneously injected into the right flank of nude mice. Tumor size was determined every 7 days by measuring the width and length of the formed tumors. The volume of the tumors was calculated with the following formula: tumor volume $=\left(\right.$ width $^{2} \times$ length $) / 2$. Nude mice were sacrificed at 35 days for further assessment of the expression of Ki67 by IHC.

\section{Statistical analysis}

All data are presented as the mean \pm standard deviation (SD) of three independent experiments. Comparisons between two groups were performed using Student's t-test. Pearson chi-square test was used to analyze the relationship among TPPP3 and clinicopathological features. Survival curves were obtained by the Kaplan-Meier method with log-rank test. A P-value $<0.05$ was considered to be statistically significant.

\section{Results}

\section{TPPP3 is highly expressed in NSCLC tumor tissues and cell lines}

Although our previous study demonstrated that TPPP3 silencing inhibited tumor growth and metastasis in vitro and in vivo [14], TPPP3 expression levels in lung tissues were unknown. First, we examined TPPP3 mRNA expression in NSCLC tissues and corresponding adjacent normal lung tissues. As shown in Figure 1A, TPPP3 mRNA expression levels were significantly upregulated in NSCLC tissue compared with adjacent normal tissue. Furthermore, TPPP3 expression was measured in the normal human bronchial cell line HBE and four NSCLC cell lines by real-time PCR and western blot. As shown in Figure 1B, TPPP3 mRNA levels were increased in NSCLC cells compared with HBE cells. Consistently, 
TPPP3 protein levels were markedly increased in NSCLC cells compared with HBE cells (Figure 1C). These results showed that TPPP3 was over-expressed in NSCLC tissues and cells.

\section{Association between TPPP3 expression and clinicopathological features of NSCLC}

To further confirm the expression of TPPP3 in NSCLC tissue samples, IHC was performed to detect TPPP3 protein levels in 76 pairs of paraffin-embedded cancer and adjacent normal tissues from patients. Representative immunohistochemical staining results showed that TPPP3 expression in cancer tissues was considerably increased compared with adjacent normal tissues (Figure 2A). The relationship between TPPP3 and clinicopathological variables is presented in Table 1. Statistical analysis showed that TPPP3 protein expression is significantly associated with tumor size, lymph node metastasis and clinical stage. In contrast, TPPP3 expression did not correlate with age, sex, tumor location and other clinicopathological characteristics. In addition, Kaplan-Meier analysis was used to analyze the difference in overall survival between patients with high and low TPPP3 expression. The survival curves showed that high TPPP3 expression was associated with short overall survival (Figure 2B), indicating that TPPP3 predicted a poor prognosis in NSCLC.

\section{TPPP3 silencing inhibited NSCLC cell growth in vitro}

To study the biological function of TPPP3, we selected two NSCLC cell lines (A549 and H1229) as cell models that have high endogenous TPPP3 expression. As shown in Figure 3A, western blot

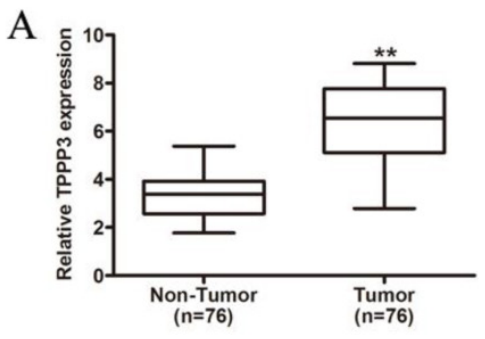

B
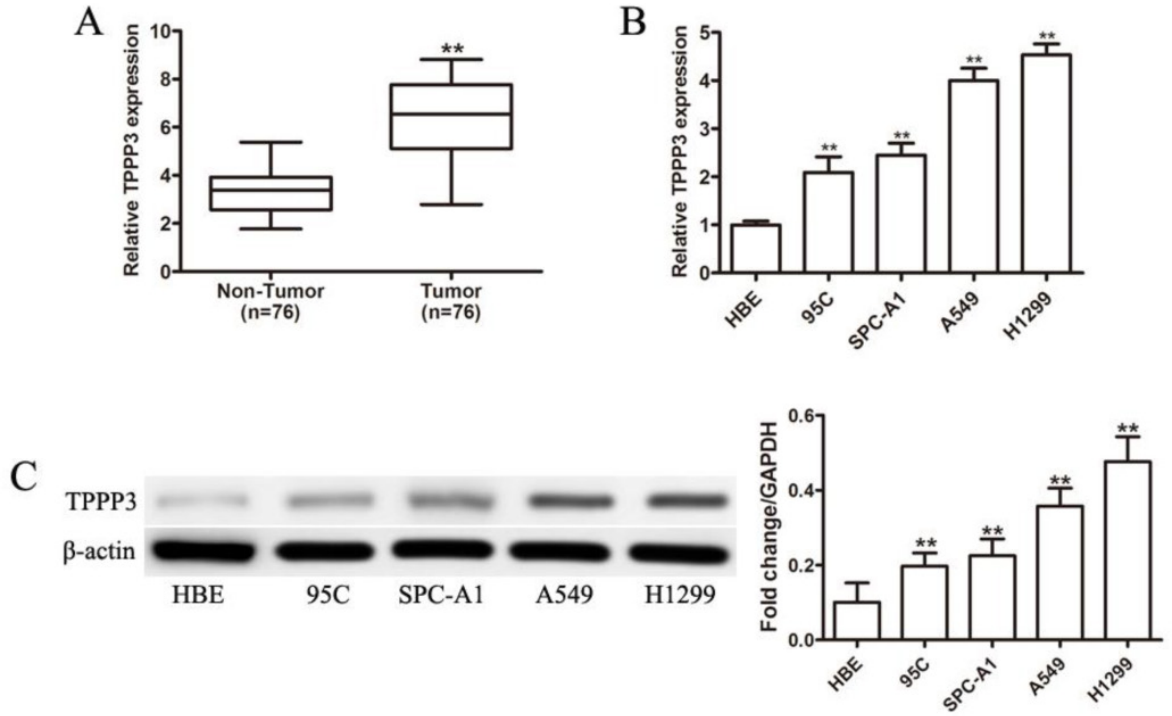

Figure 1. TPPP3 expression is significantly upregulated in primary human lung cancer and NSCLC cell lines. (A) TPPP3 mRNA expression in 76 pairs of NSCLC tumor tissues and adjacent normal lung tissues was determined using real-time PCR. (B) Real-time PCR was performed to assess TPPP3 mRNA levels in NSCLC cells and HBE. (C) Western blot analysis showed TPPP3 protein expression in a panel of human NSCLC cell lines and HBE. Data were quantified via densitometric analyses from at least three independent experiments. $* * P<0.01$.

indicated that two shRNA viruses were able to efficiently suppress endogenous TPPP3 expression in A549 and H1229 cells. shRNA2 of TPPP3 was the most highly functional shRNA and was used in the following experiments. Knockdown of TPPP3 significantly suppressed cell viability by CCK- 8 assay (Figure 3B), and clone formation was also inhibited compared with the scrambled control shRNA (Figure 3C). These results showed that TPPP3 plays an important role in NSCLC cell proliferation.

Table 1. Patient clinicopathological features and TPPP3 expression.

\begin{tabular}{llll}
\hline Variables & \multicolumn{3}{c}{ TPPP3 expression level } \\
\cline { 2 - 4 } & Low N=38 & High N=38 & P-value \\
\hline Gender & 16 & 20 & 0.4910 \\
Female & 22 & 18 & \\
Male & & & \\
Age, & 17 & 13 & 0.4818 \\
$\leq 60$ & 21 & 25 & \\
$>60$ & & & \\
Location & 18 & 19 & 1.0000 \\
Left & 20 & 19 & \\
Right & & & \\
Histology & 29 & 24 & 0.3180 \\
Adenocarcinoma & 9 & 14 & \\
Squamous cell carcinoma & & & \\
Tumor Size (cm) & 28 & 14 & 0.0010 \\
$\leq 3$ & 9 & 24 & \\
$>3$ & & & \\
Lymph node metastasis & 11 & 23 & 0.0107 \\
Yes & 27 & 15 & \\
No & & & \\
Clinical Stage & 31 & 18 & 0.0036 \\
I-II & 7 & 20 & \\
III & & &
\end{tabular}


Figure 2. High TPPP3 expression is associated with poor prognosis. (A) Typical TPPP3 staining in lung cancer and adjacent normal lung tissues. Left, magnification 50X; Right, magnification 200X. (B) The Kaplan-Meier curves showed that patients with high TPPP3 expression had shorter overall survival than patients with low TPPP3 expression.

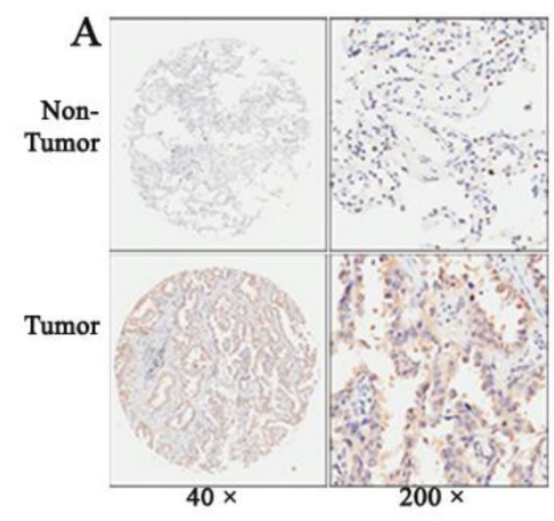

B

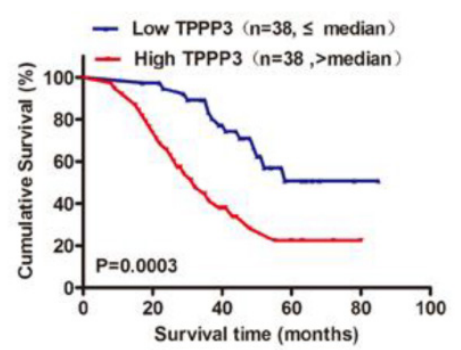

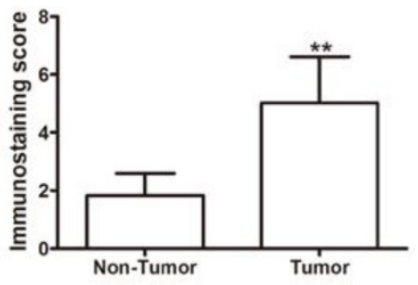

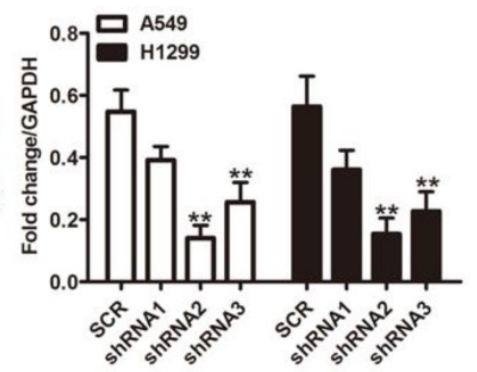

B

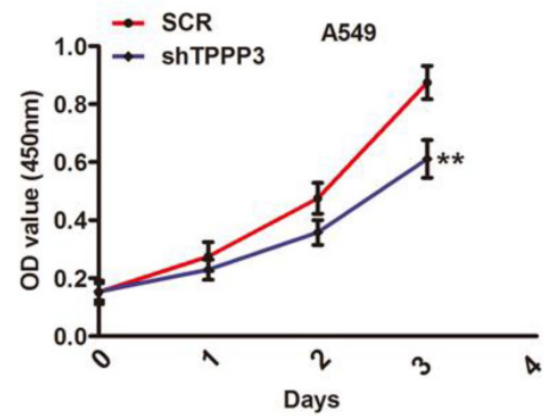

C

A549

H1299

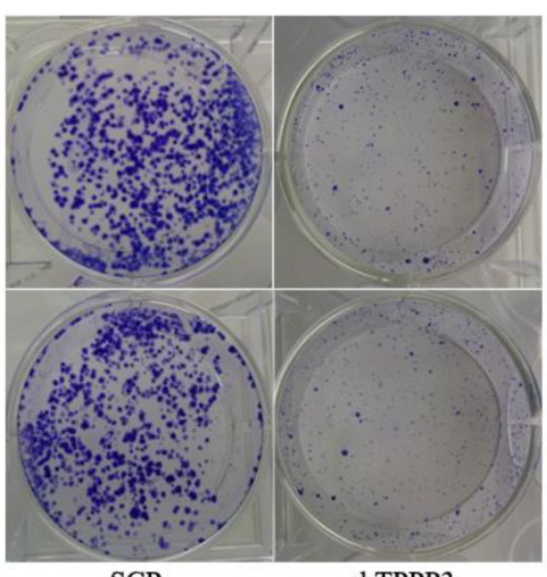

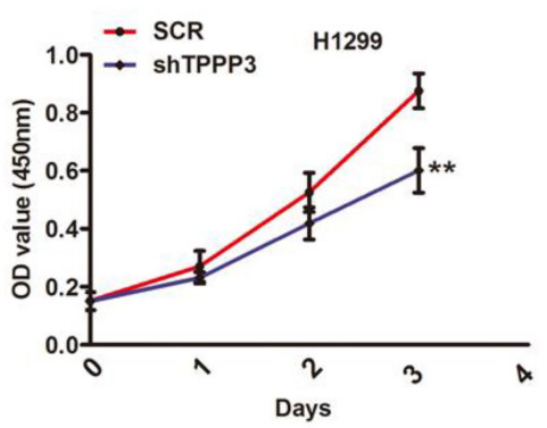

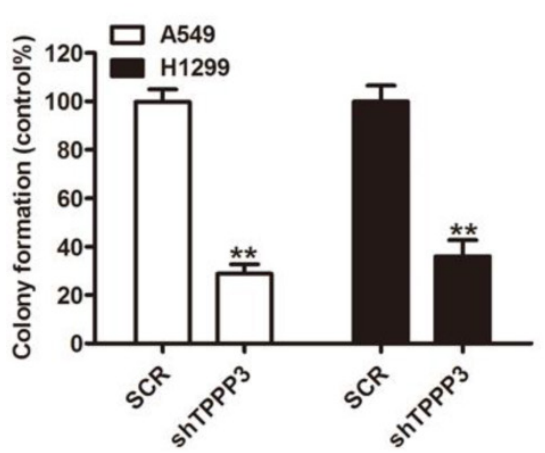

Figure 3. Knockdown of TPPP3 inhibited NSCLC cell proliferation. (A) TPPP3 expression in A549 and H1299 cells transduced with SCR or different shTPPP3 sequences as assessed by western blot. Data were quantified via densitometric analyses from at least three independent experiments. (B) A549 and $\mathrm{H} 1299$ cell viability was measured using the CCK-8 assay. (C) Colonies were counted in A549 and $\mathrm{H} 1299$ cells after transduction with $\mathrm{SCR}$ or ShTPPP3 at 14 days. Each bar represents the relative colony number and SD of 3 wells. $* * P<0.01$. 


\section{Knockdown of TPPP 3 induced cell apoptosis and cell cycle arrest}

Flow cytometric analysis of apoptosis and the cell cycle was performed to investigate the mechanism by which depletion of TPPP3 inhibits cell proliferation. Annexin V staining showed that TPPP3 silencing resulted in increased apoptotic levels both in A549 and H1229 cells (Figure 4A). In cells infected with shTPPP3, the number of cells in $S$ phase was significantly decreased, whereas G0/G1 phase was notably increased compared with cells infected with scrambled shRNA (Figure 4B). To elaborate the molecular mechanism by which TPPP3 regulated cell proliferation in NSCLC cells, we detected a number of important proteins and signaling pathways related to tumorigenesis. As shown in Figure 4C, depletion of TPPP3 effectively decreased the levels of p-AKT, p-STAT3, Survivin, CyclinD1, and BCL-2 both in A549 and H1229 cells. These results suggested that TPPP3 plays an important role in the regulation of apoptosis and the cell cycle in lung cancer cells.

\section{Inhibition of TPPP3 significantly inhibits tumorigenicity in nude mice}

Given that our results indicated that TPPP3 knockdown inhibited cell proliferation by inducing cell apoptosis and cell cycle arrest in vitro, we sought to determine the effect of TPPP3 on tumorigenicity in vivo. H1229 cells infected with scrambled or shTPPP3 were subcutaneously injected into the right flank of nude mice. A significant reduction in tumor volume was recorded in the shTPPP3 group compared with the SCR group at 5 weeks (Figure 5A). Compared with the SCR group, the tumor volume and weight were significantly reduced in the group treated with shTPPP3 (Figure 5B and 5C). In addition, the proliferation of cell-associated nuclear antigen Ki67 was determined in the xenograft tumor tissues. As shown in Figure 5D, Ki67 expression was markedly attenuated in the group treated with shTPPP3. In summary, these data indicated that knockdown of TPPP3 inhibited tumor growth in vivo.
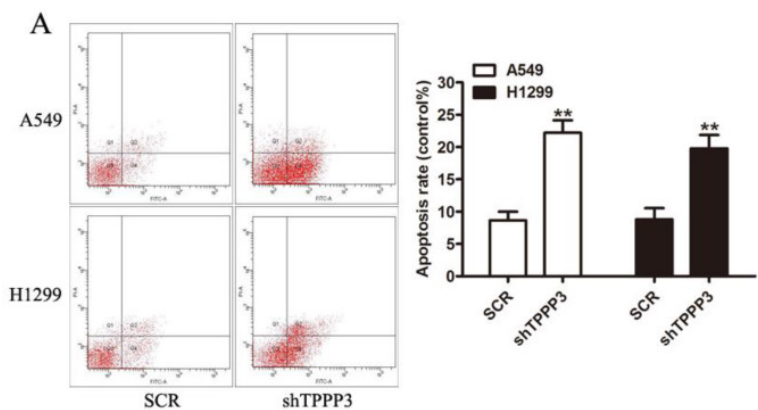

B
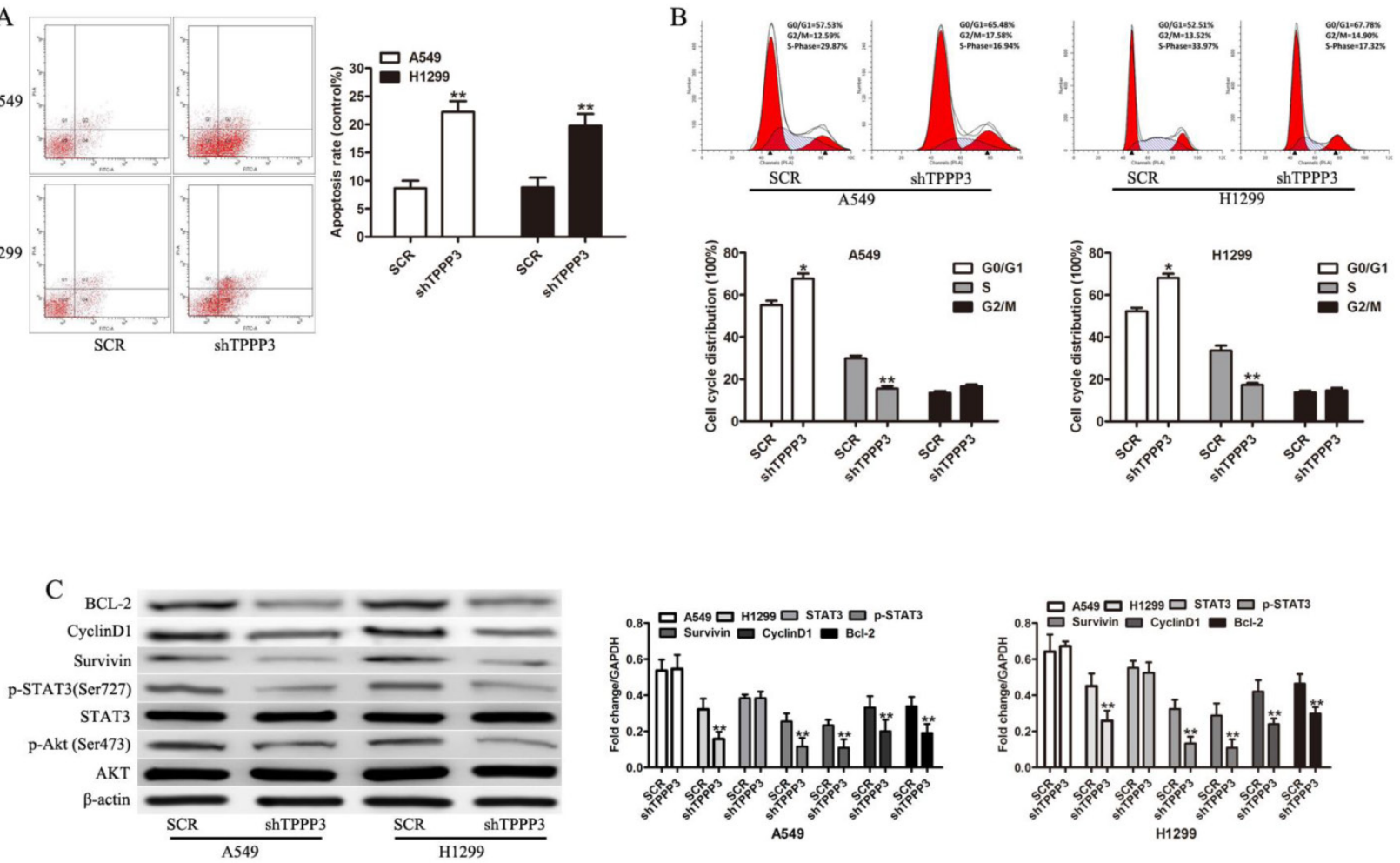

Figure 4. The effects of TPPP3 on cell apoptosis and cell cycle. (A) Cell death was assessed by Annexin V staining and flow cytometry. (B) Cell cycle analysis was performed by flow cytometry after knockdown of TPPP3 in A549 and H1299 cells. (C) Western blot showed the expression of AKT, P-AKT, P-STAT3, STAT3, Survivin, CyclinDI, and BCL-2 in A549 and H1299 cells after TPPP3 silencing. Data were quantified via densitometric analyses from at least three independent experiments. ${ }^{*} \mathrm{P}<0.01$. 

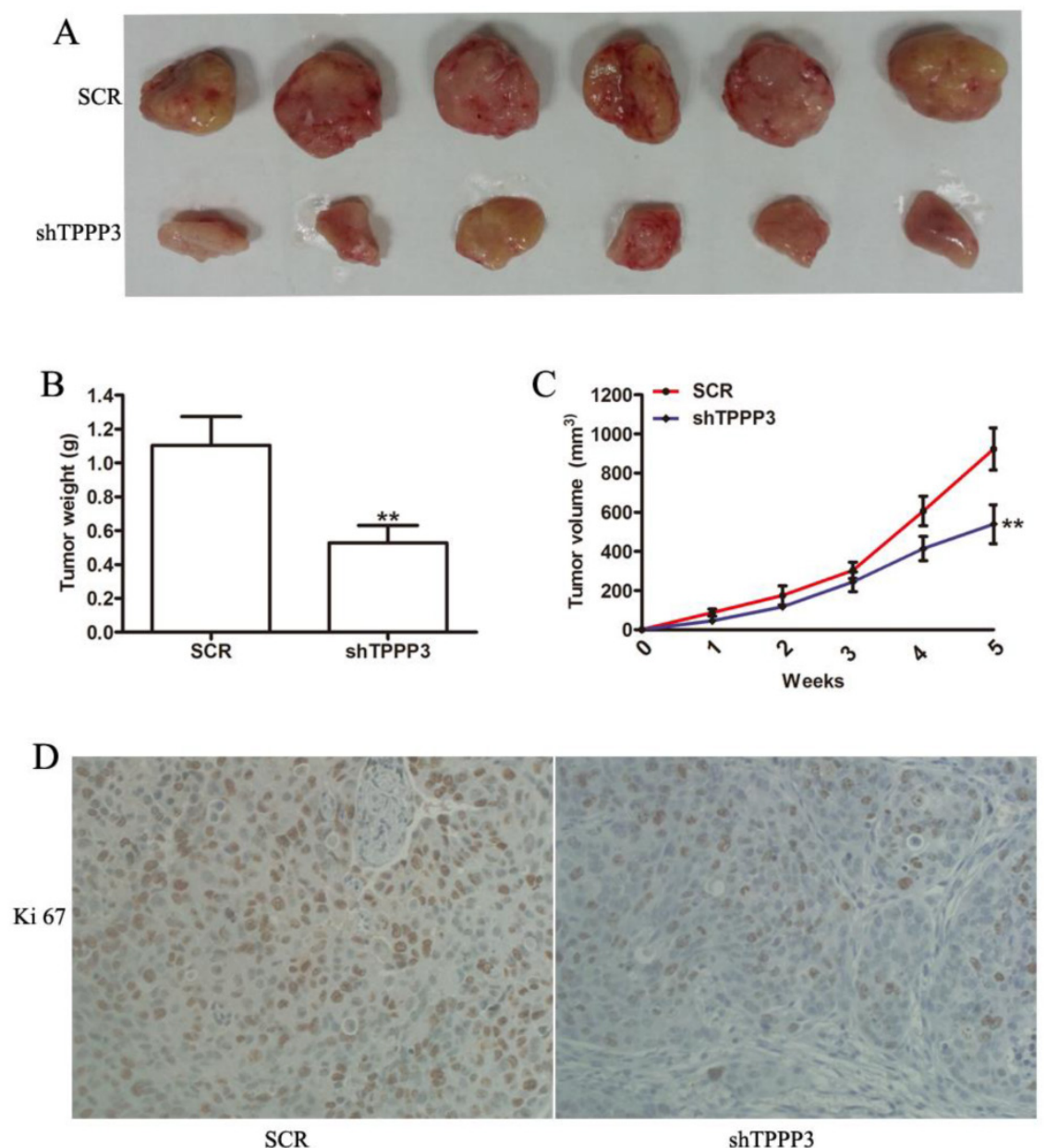

Figure 5. Knockdown of TPPP3 in H1299 cells suppressed tumor growth in vivo. (A) Representative photographs of tumors following the subcutaneous injection of H1299 cells with or without TPPP3 silencing in nude mice at 35 days. (B and C) Tumor weight and growth curves were recorded. (D) Representative images of Ki67 in xenograft tumor tissues from the SCR or shTPPP3 groups. ${ }^{* *} \mathrm{P}<0.01$.

\section{Discussion}

Studies have shown that some genes are dysfunctional and act as oncogenes or tumor suppressors in NSCLC [16-18]. Our previous studies demonstrated that TPPP3 silencing could inhibit the growth of HeLa and Lewis lung carcinoma cells [5,14]. The increase in TPPP3 was noted by 2D-PAGE, thus revealing that TPPP3 is a new biomarker of potential diagnostic or therapeutic utility in NSCLC [13]. In our present study, we found that TPPP3 expression was increased in NSCLC tissues and cell lines. Furthermore, the relationship between TPPP3 protein expression and clinical characteristics indicated that high TPPP3 expression was positively associated with tumor size, lymph node metastasis, clinical stage and poor prognosis. Moreover, we explored the biological functions of TPPP3 on cell growth, apoptosis, and the cell cycle and found that TPPP3 plays a key role in enhancing cell proliferation, inhibiting cell apoptosis, and accelerating the cell cycle. These results are consistent with our recently published study that demonstrated that knockdown of TPPP3 by siRNA inhibited tumor growth and metastasis [14].

Inhibition of cell proliferation is typically related to induction of apoptosis and cell cycle arrest [19-21]. Our results showed that the growth inhibitory effect of TPPP3 was related to the induction of apoptosis and cell cycle arrest. To elucidate the possible mechanism involved in cell proliferation, a number of important moleculars associated with tumorigenesis were detected. AKT and STAT3 participate in the growth and anti-apoptotic property of cancer cells [22-24]. It is valuable to discover whether TPPP3 influences the signaling cascade of the AKT and STAT3 pathways, which are involved in cell apoptosis and the cell cycle $[25,26]$. As expected, downregulation of TPPP3 lead to a decrease in phospho-Akt and phospho-STAT3 as well as their downstream genes, such as Survivin, CyclinD1, and BCL-2.

In summary, our study demonstrated for the first time that TPPP3 was increased in lung tissues and contributed to the progression of NSCLC through 
the Akt and STAT3 signaling pathway. Our data reveal new insights into the mechanism responsible for the progression of NSCLC, and more detailed molecular mechanisms of TPPP3 in lung tumor progression require further research.

\section{Acknowledgments}

This work was supported by grants from the National Natural Science Foundation of China (No. 81101762, 81471057, 81402288, 81270902) and Promotive research fundation for excellent young and middle-aged scientisits of Shandong Province (No. BS2015YY028, BS2014YY040).

\section{Conflicts of interest}

The authors declare no conflict of interest.

\section{References}

1 Ferlay J, Shin HR, Bray F, Forman D, Mathers C, Parkin DM: Estimates of worldwide burden of cancer in 2008: GLOBOCAN 2008. Int J Cancer 2010;127:2893-2917.

2 Ballatori E, Fatigoni S, Roila F: Treatment of lung cancer. N Engl J Med 2009;361:2485-2487.

3 Torre LA, Bray F, Siegel RL, Ferlay J, Lortet-Tieulent J, Jemal A: Global cancer statistics, 2012. CA Cancer J Clin 2015;65:87-108.

4 Kadavath H, Hofele RV, Biernat J, Kumar S, Tepper K, Urlaub H, Mandelkow E, Zweckstetter M: Tau stabilizes microtubules by binding at the interface between tubulin heterodimers. Proc Natl Acad Sci U S A 2015;112:7501-7506.

5 Zhou W, Wang X, Li L, Feng X, Yang Z, Zhang W, Hu R: Depletion of tubulin polymerization promoting protein family member 3 suppresses HeLa cell proliferation. Mol Cell Biochem 2010;333:91-98.

6 Wieczorek M, Bechstedt S, Chaaban S, Brouhard GJ: Microtubule-associated proteins control the kinetics of microtubule nucleation. Nat Cell Biol 2015;17:907-916.

7 Vincze O, Tokesi N, Olah J, Hlavanda E, Zotter A, Horvath I, Lehotzky A, Tirian L, Medzihradszky KF, Kovacs J, Orosz F, Ovadi J: Tubulin polymerization promoting proteins (TPPPs): Members of a new family with distinct structures and functions. Biochemistry-Us 2006;45:13818-13826.

8 Hu RM, Han ZG, Song HD, Peng YD, Huang QH, Ren SX, Gu YJ, Huang CH, Li YB, Jiang CL, Fu G, Zhang QH, Gu BW, Dai M, Mao YF, Gao GF, Rong R, Ye M, Zhou J, Xu SH, Gu J, Shi JX, Jin WR, Zhang CK, Wu TM, Huang GY, Chen $Z$, Chen MD, Chen JL: Gene expression profiling in the human hypothalamus-pituitary-adrenal axis and full-length cDNA cloning. Proc Natl Acad Sci U S A 2000;97:9543-9548.

9 Orosz F: A new protein superfamily: TPPP-like proteins. PLoS One 2012;7:e49276.

10 Orosz F: On the tubulin polymerization promoting proteins of zebrafish. Biochem Biophys Res Commun 2015;457:267-272.

11 Tirian L, Hlavanda E, Olah J, Horvath I, Orosz F, Szabo B, Kovacs J, Szabad J, Ovadi J: TPPP/p25 promotes tubulin assemblies and blocks mitotic spindle formation. Proc Natl Acad Sci U S A 2003;100:13976-13981.

12 Staverosky JA, Pryce BA, Watson SS, Schweitzer R: Tubulin polymerization-promoting protein family member 3, Tppp3, is a specific marker of the differentiating tendon sheath and synovial joints. Dev Dyn 2009;238:685-692.

13 Pastor MD, Nogal A, Molina-Pinelo S, Melendez R, Salinas A, Gonzalez DLPM, Martin-Juan J, Corral J, Garcia-Carbonero R, Carnero A, Paz-Ares L: Identification of proteomic signatures associated with lung cancer and COPD. J Proteomics 2013;89:227-237.

14 Zhou W, Li J, Wang X, Hu R: Stable knockdown of TPPP3 by RNA interference in Lewis lung carcinoma cell inhibits tumor growth and metastasis. Mol Cell Biochem 2010;343:231-238.

15 Chen J, Gu J, Feng J, Liu Y, Xue Q, Ni T, Wang Z, Jia L, Mao G, Ji L: TAB3 overexpression promotes cell proliferation in non-small cell lung cancer and mediates chemoresistance to CDDP in A549 cells via the NF-kappaB pathway. Tumour Biol 2015

16 Qiao W, Han Y, Jin W, Tian M, Chen P, Min J, Hu H, Xu B, Zhu W, Xiong L, Lin Q: Overexpression and biological function of TMEM48 in non-small cell lung carcinoma. Tumour Biol 2015

17 Dai M, Lu JJ, Guo W, Yu W, Wang Q, Tang R, Tang Z, Xiao Y, Li Z, Sun W, Sun $X$, Qin $Y$, Huang W, Deng WG, Wu T: BPTF promotes tumor growth and predicts poor prognosis in lung adenocarcinomas. Oncotarget 2015;6:33878-33892.

18 Sakashita S, Sakashita M, Sound TM: Genes and pathology of non-small cell lung carcinoma. Semin Oncol 2014;41:28-39.
19 Chen L, Tian H, Li M, Ge C, Zhao F, Zhang L, Li H, Liu J, Wang T, Yao M, Li J: Derivate isocorydine inhibits cell proliferation in hepatocellular carcinoma cell lines by inducing G2/M cell cycle arrest and apoptosis. Tumour Biol 2015

20 Mawatari T, Ninomiya I, Inokuchi M, Harada S, Hayashi H, Oyama K, Makino I, Nakagawara H, Miyashita T, Tajima H, Takamura H, Fushida S, Ohta T: Valproic acid inhibits proliferation of HER2-expressing breast cancer cells by inducing cell cycle arrest and apoptosis through Hsp70 acetylation. Int J Oncol 2015;47:2073-2081.

21 Gong Y, Qiu W, Ning X, Yang X, Liu L, Wang Z, Lin J, Li X, Guo Y: CCDC34 is up-regulated in bladder cancer and regulates bladder cancer cell proliferation, apoptosis and migration. Oncotarget 2015;6:25856-25867.

22 Wu Y, Xu X, Miao X, Zhu X, Yin H, He Y, Li C, Liu Y, Chen Y, Lu X, Wang Y, He S: Sam68 regulates cell proliferation and cell adhesion-mediated drug resistance (CAM-DR) via the AKT pathway in non-Hodgkin's lymphoma. Cell Prolif 2015;48:682-690

23 Chen J, Bai M, Ning C, Xie B, Zhang J, Liao H, Xiong J, Tao X, Yan D, Xi X, Chen X, Yu Y, Bast RC, Zhang Z, Feng Y, Zheng W: Gankyrin facilitates follicle-stimulating hormone-driven ovarian cancer cell proliferation through the PI3K/AKT/HIF-1alpha/cyclin D1 pathway. Oncogene 2015

24 Silva JM, Bulman C, McMahon M: BRAFV600E cooperates with PI3K signaling, independent of AKT, to regulate melanoma cell proliferation. Mol Cancer Res 2014;12:447-463.

25 Shen G, Rong X, Zhao J, Yang X, Li H, Jiang H, Zhou Q, Ji T, Huang S, Zhang J, Jia H: MicroRNA-105 suppresses cell proliferation and inhibits PI3K/AKT signaling in human hepatocellular carcinoma. Carcinogenesis 2014;35:2748-2755.

26 Li WF, Dai H, Ou Q, Zuo GQ, Liu CA: Overexpression of microRNA-30a-5p inhibits liver cancer cell proliferation and induces apoptosis by targeting MTDH/PTEN/AKT pathway. Tumour Biol 2015. 